\title{
THE VALUE SEMIGROUPS OF PRIME DIVISORS OF THE SECOND KIND IN 2-DIMENSIONAL REGULAR LOCAL RINGS
}

\author{
SUNSOOK NOH
}

\begin{abstract}
In this paper, it is shown that the value semigroup of a prime divisor of the second kind on a 2-dimensional regular local ring is symmetric. Further, a necessary and sufficient condition for two prime divisors of the second kind on a 2-dimensional regular local ring to have the same value semigroup is obtained.
\end{abstract}

\section{INTRODUCTION}

Let $(R, m, k)$ be a 2-dimensional regular local ring (RLR for short) with maximal ideal $m$ and residue field $k$. Let $w$ be a prime divisor of the quotient field $K$ of $R$. Then $w$ is called a prime divisor of the second kind on $R$ if the valuation ring $W$ of $w$ birationally dominates $R$ and $\operatorname{tr} \operatorname{deg}_{k}(k(w))=1$, where $k(w)$ denotes the residue field of $W$. In Appendix 5 of [ZS], Zariski set up a one-to-one correspondence between the set of prime divisors of the second kind on $R$ and the set of simple integrally closed $m$-primary ideals of $R$. Recall that an ideal is simple if it is not the unit ideal and has no nontrivial factorizations.

To explain this correspondence, we first briefly discuss quadratic transformations of 2-dimensional RLRs and transforms of integrally closed ideals (for more details we refer the reader to Appendix 5 in [ZS] and also [L2, H]).

Let $m=(x, y)$; for a maximal ideal $N$ of $R[m / x]$ containing $m R[m, x]$, $R[m, x]_{N}$ is a 2-dimensional RLR with maximal ideal $N R[m / x]_{N}$. Let $R_{1}=$ $R[m / x]_{N}$. Then $R_{1}$ is called a first quadratic transformation of $R$. If $I$ is an ideal of $R$ of order $r$ contracted from $R[m / x]$, then we may write $I R[m / x]=x^{r} I^{\prime}$, and $I^{\prime}$ is called the transform of $I$ in $R[m / x]$. Let $I_{1}=I_{N}^{\prime}$. Then $I_{1}$ is called the transform of $I$ in $R_{1}$. If $J$ is an ideal of $R[m / x]$ of height 2, then since $R[m / x]=\bigcup m^{n} / x^{n}$ for $n \geq 0$, it follows that $J$ $=\left(r_{1} / x^{n}, \ldots, r_{t} / x^{n}\right) R[m / x]$ for some $n$ and $r_{i} \in R$, that is, $x^{n} J=$ $\left(r_{1}, \ldots, r_{t}\right) \cdot R[m / x]$ is extended from $R$. Let $a$ be the least positive integer such that $x^{a} J$ is extended from $R$. Then $x^{a} J \cap R$ is called the inverse transform of $J$.

Zariski proved that $I$ is integrally closed and contracted from $R[m / x]$ if and only if $I^{\prime}$ is integrally closed in $R[m / x]$. He also proved that if $I$ is a simple integrally closed $m$-primary ideal which is contracted from $R[m / x]$, then $I^{\prime}$ is simple, and $I^{\prime}$ is contained in a unique maximal ideal $N$ of $R[m / x]$

Received by the editors April 2, 1990 and, in revised form, November 28, 1990.

1980 Mathematics Subject Classification (1985 Revision). Primary 13A18, 13B20, 13F30, 13 H05. 
and $I_{1}$ is simple in $R_{1}=R[m / x]_{N}$. Conversely, the inverse transform of a simple (integrally closed $m$-primary) ideal is simple.

An ideal $I$ is said to be contracted if there is some $x \in m \backslash m^{2}$ such that $I R[m / x] \cap R=I$. Zariski defined the characteristic form $c(I)$ of an $m$ primary ideal $I$ of order $r$ to be the greatest common divisor of the elements in $\left(I+m^{r+1}\right) / m^{r+1}$ in the UFD $\mathrm{gr}_{m}(R)=R / m \oplus m / m^{2} \oplus \ldots$ [ZS, p. 363, Appendix 5]. Let $x^{*}$ be the image of $x$ in $\operatorname{gr}_{m}(R)$; then for an $m$-primary ideal $I, x^{*} \nmid c(I)$ if and only if $I$ is contracted from $R[m / x]$. Now we can explain the one-to-one correspondence between simple integrally closed $m$-primary ideals and the prime divisors of the second kind.

For a simple $m$-primary integrally closed ideal $I$, there exists a unique finite quadratic sequence of 2-dimensional RLRs

$$
\left(R=R_{0}, m_{0}\right) \subset\left(R_{1}, m_{1}\right) \subset \cdots \subset\left(R_{n}, m_{n}\right),
$$

where the ideal transform of $I$ in $R_{n}$ is the maximal ideal $m_{n}$. Then the $m_{n}$ adic order valuation is the prime divisor of the second kind associated to $I$. On the other hand, for each prime divisor $w$ with the associated valuation ring $(W, m(W), k(w))$, there exists a unique finite quadratic sequence of RLRs

$$
\left(R=R_{0}, m=m_{0}\right) \subset\left(R_{1}, m_{1}\right) \subset \cdots \subset\left(R_{n}, m_{n}\right)
$$

such that $w$ is the $m_{n}$-adic order valuation. Then the inverse transform of $m_{n}$ in $R$ is a simple integrally closed $m$-primary ideal of $R$ which is uniquely associated to $w$.

From now on, by a prime divisor we mean a prime divisor of the second kind. A numerical semigroup $H$ is a subsemigroup of the nonnegative integers $N$ under addition with $0 \in H$ and $n+N \subseteq H$ for some integer $n$. The least integer $c \in H$ with that property is called the conductor element of $H$. Also $H$ is called symmetric if there exists an integer $m$ such that $z \in H$ if and only if $m-z \notin H$ for every integer $z$. For a prime divisor $w$ of $R$, let $w(R)=\{w(r) \mid r \in R\}$. Then $w(R)$ is a numerical semigroup and is called the value semigroup of $w$ on $R$. In this paper we study some other properties of the prime divisors of the second kind of a 2-dimensional RLR which were not discussed in Appendix 5 of [ZS]. In [Hos], Hoskin showed that a valuation $v$ dominating $R$ with the residual transcendence degree 0 over $R$ has a symmetric value semigroup $v(R)$ on $R$ [Hos, Lemma 5.2]. In this paper we primarily investigate the value semigroups of the prime divisors of the second kind.

Specifically, in $\S 1$ we prove the following theorem by using a nice theorem of Kunz [K1] on the value semigroup of a 1-dimensional local analytically irreducible domain.

Theorem 1. Let $(R, m, k)$ be a 2-dimensional $R L R$ with algebraically closed residue field $k$. Let $w$ be a prime divisor of the second kind on $R$. Then the value semigroup $w(R)$ on $R$ is symmetric.

Once we prove the above theorem (in $\S 1$ ), we shall be interested in the integer $c$ in $w(R)$ which is the conductor element of the value semigroup $w(R)$. The ideal $C=\{r \in R \mid w(r) \geq c\}$ of $R$ is called the conductor ideal of $w$. With respect to the conductor element and the conductor ideal of a prime divisor, Lipman [L3] has obtained many nice formulae in terms of the point basis of 
an integrally closed ideal which is associated to the given prime divisor. We introduce some of them in $\S 2$. Conductors of the value semigroups of plane algebroid curves are also studied in $[\mathrm{Az}, \mathrm{Hos}, \mathrm{K} 2]$ in terms of characteristic pairs, Apéry sequence, and multiplicity sequence.

Let $w$ and $v$ be two different prime divisors of $R$, and let $c(w)$ and $c(v)$ be the conductors of $w$ and $v$ respectively. In $\S 2$, we shall study conditions under which these two conductors are the same and also under which the two value semigroups $w(R)$ and $v(R)$ on $R$ are the same. In [Az], Azevedo showed that the value semigroups of two plane algebroid curves are equal if and only if their multiplicity sequences are equal. By showing that the point basis of a simple integrally closed ideal which is associated to the given prime divisor gives the multiplicity sequence of a 1-dimensional analytically irreducible Gorenstein local domain, we find special cases where those two value semigroups are equal in Theorem 2. Two ideals $I \supset J$ of $R$ are said to be adjacent if $I / J \simeq R / m$.

Theorem 2. Let $(R, m, k)$ be a 2-dimensional $R L R$ with algebraically closed residue field $k$. Let $w$ and $v$ be two prime divisors of the second kind on $R$ which are associated to two adjacent simple m-primary integrally closed ideals. Then $w(R)=v(R)$, and hence $c(w)=c(v)$.

By using Theorems 1 and 2 we can generalize Theorem 2; we obtain the following necessary and sufficient condition for two prime divisors $v$ and $w$ to have the same value semigroups. (The characterization is in terms of the point bases of simple integrally closed ideals $I$ and $J$ associated to $v$ and $w$. See $\S 2$ for the definition of the point basis $B(I)$ of an ideal $I$, which is due to Lipman.)

Theorem 3. Let $(R, m, k)$ be a 2-dimensional $R L R$ with algebraically closed residue field $k$. Let $v$ and $w$ be prime divisors of the second kind on $R$ which are associated to two simple m-primary integrally closed ideals $I$ and $J$ respectively. Let

$$
B(I)=\left\{s_{i}\right\}_{i=1}^{p} \text { and } B(J)=\left\{t_{j}\right\}_{j=1}^{q},
$$

and suppose $s_{i}=1$ iff $i \geq n$ and $t_{j}=1$ iff $j \geq h$. Then $w(R)=v(R)$ if and only if $h=n$ and $s_{i}=t_{i}$ for $i=0,1, \ldots, n$.

Since we heavily use Zariski's theory of 2-dimensional RLRs it seems hard to generalize the main theorems of this paper. We present some examples which illustrate the theorems and indicate restrictions on possible generalizations. We also raise some questions about possible generalizations of the theorems which are proved in this paper.

\section{SyMmetric VAlue SEMigroup}

In this section we are going to prove Theorem 1, which was stated in the Introduction. Before we list and prove all the necessary requirements, we first recall a few definitions and notation. The order $o(I)$ of an ideal $I$ of a local ring $(R, m)$ is $r$ if $I \subseteq m^{r}$, but $I \nsubseteq m^{r+1}$. For an element $a$ in a ring $R, a$ is integral over an ideal $I$ of $R$ if $a$ satisfies the equation

$$
x^{n}+a_{1} x^{n-1}+\cdots+a_{n-1} x+a_{n}=0
$$

for some $a_{i} \in I^{i}, i=1,2, \ldots, n$. The set of all the elements integral over $I$ is called the integral closure of $I$ and is denoted by $\bar{I}$. For ideals $J \subseteq I$, 
$J$ is called a reduction of $I$ if $I^{n+1}=J I^{n}$ for some $n \geq 1$. The number of elements in a basis of an ideal of a local ring is denoted by $\mu(I)$, and $\lambda(M)$ denotes the length of an $R$-module $M$. The multiplicity of a local ring $R$ or of an ideal $I$ of a local ring is denoted by $e(R)$ or $e(I)$. For a valuation $v$ of the quotient field $K$ of $R$ which has nonnegative values on $R$, an ideal $I$ is said to be a $v$-ideal if $I V \cap R=I$, where $V$ is the valuation ring of $v$.

Now we introduce the theorem of Kunz which is essential to the proof of our main theorem.

Theorem 1.1 (Kunz [K1]). Let $A$ be a 1-dimensional analytically irreducible Noetherian local ring. Let $\bar{A}$ be the integral closure of $A$ in $K=q . f .(A), a$ discrete valuation ring, and let $v$ be the corresponding valuation on $K$. Assume that $A$ and $\bar{A}$ have the same residue field. Then $A$ is Gorenstein if and only if the value semigroup $v(A)$ on $A$ is symmetric.

We also state two remarks on prime divisors of 2-dimensional RLRs.

Remark 1.2 (Zariski [ZS, Appendix 5]). Let $(R, m, k)$ be a 2-dimensional RLR and let $w$ be a prime divisor of $R$ associated to the simple integrally closed ideal $J$. Let $(a, b)$ be a minimal reduction of $J$. If $k$ is algebraically closed, then $k(w)$ is purely transcendental over $k$ and is generated by the image of $a / b$.

Remark 1.3 (Heinzer [HS, Remark 3.6]). Let $(R, m, k)$ be a 2-dimensional RLR and let $w$ be a prime divisor of $R$. Let $a$ and $b$ be elements of $R$ such that $w(a)=w(b)$ and the image of $a / b$ in $k(w)$ is purely transcendental over $k$. Then $w$ is one of the Rees valuations of $(a, b)$.

Both of the above remarks are proved in [HS]. Some general references for the theory of Rees valuations are [M, R]. After Zariski's foundation of the theory of integrally closed ideals in 2-dimensional RLRs, Lipman proved many things on multiplicities of integrally closed ideals and values of integrally closed ideals with respect to prime divisors in 2-dimensional RLRs in [L1, L2, L3] as well as in many other unpublished handwritten notes. From among these, we state two theorems that we need at the beginning of the proof of Theorem 1 .

Theorem 1.4 (Lipman [L1, (21.4)]). Let $w$ and $v$ be two prime divisors of a 2-dimensional $R L R(R, m, k)$ which are associated to simple integrally closed ideals $J$ and $I$ respectively. Then

$$
v(J) \Delta(v)=w(I) \Delta(w),
$$

where $\Delta(v)=\left[k_{v}: k\right], k_{v}=$ the algebraic closure of $k$ in $k(v)$.

Theorem 1.5 (Lipman [L1, (23.2); L2, (1.11) and (3.8)]). Let I be an integrally closed ideal in a 2-dimensional $R L R(R, m, k)$, and let $I=I_{1}^{k_{1}} I_{2}^{k_{2}} \cdots I_{n}^{k_{n}}$ be its unique factorization into a product of simple integrally closed ideals $I_{1}, \ldots, I_{n}$. Let $v_{i}$ be the prime divisor associated to $I_{i}$ for $i=1, \ldots, n$. Then

$$
e(I)=\sum_{i=1}^{n} k_{i} v_{i}(I) \Delta\left(v_{i}\right)
$$

Now we are ready to prove the main theorem of this section. 
Proof of Theorem 1. Let $J$ be a simple integrally closed ideal corresponding to $w$. Hence, $w$ is the only Rees valuation of $J$. Let $o(J)=r$ and $e(J)=e$. Since $w(m)=o(J)$ by Theorem 1.4 and $w(J)=e(J)$ by Theorem 1.5, $e$ and $r$ are in $w(R)$. Let $W^{\prime}$ be a composite of $W$ and a valuation ring of $k(w)$, with corresponding valuation $w^{\prime}$, such that $k\left(w^{\prime}\right) \cong R / m$. Since $J \subseteq$ $J W^{\prime} \cap R \subseteq J W \cap R=J, J$ is also a $w^{\prime}$-ideal. Hence, we get the chain of $w^{\prime}$ ideals from $J$ to $m$. Since $J$ is simple, this is also the chain of $w$-ideals from $J$ to $m$ (cf. [Z, Theorem 6.2; No, Theorem A.2]). Furthermore, each ideal in this chain is adjacent to the next by [Z, Theorem 1]. Since $J$ is a $w$-ideal, $J: m$ is also a $w$-ideal. But $\lambda((J: m) / J)=r$ (cf. [HS, Theorem 2.1]) implies $w(J: m) \leq e-r$, and $m(J: m) \subseteq J$ implies $w(J: m) \geq e-r$. Therefore, $w(J: m)=e-r$. Since the sequence of $w$-ideals from $J$ to $J: m$ is saturated (i.e., each is adjacent to the next), we have $e, e-1, \ldots, e-r \in w(R)$. Since $r \in w(R)$, we have for all $n \geq e-r$ that $n \in w(R)$. Let $(a, b)$ be a minimal reduction of $J$ and consider $R[a / b]$. Let $f$ be an element of $R[a / b]$. Write

$$
f=r_{0}+r_{1}(a / b)+\cdots+r_{n}(a / b)^{n},
$$

where $r_{i} \in R$. Then $w(f) \geq \min \left\{w\left(r_{j}\right)\right\}$ since $w(a)=w(b)$. If $w(f) \geq e-r$, then $w(f) \in w(R)$ since $n \in w(R)$ for all $n \geq e-r$. Suppose $w(f)<e-r$. Then we prove the following claim.

Claim. $w(f)=\min \left\{w\left(r_{j}\right)\right\}$.

Proof of claim. Let $\min \left\{w\left(r_{j}\right)\right\}=w\left(r_{i}\right)$ for some $i$. Suppose $w(f)>w\left(r_{i}\right)$. Then $w\left(r_{i}\right)<w(f)<e-r$ implies $w\left(r_{i}\right)<w(J)$. There exists some $r_{j}$ such that $w\left(r_{j}\right)=w\left(r_{i}\right)$ since if $w\left(r_{j}\right)>w\left(r_{i}\right)$ for all $j \neq i$, then $w(f)=$ $\min \left\{w\left(r_{j}\right)\right\}=w\left(r_{i}\right)$, which is a contradiction. Hence, $w\left(r_{j}\right)=w\left(r_{i}\right)$ for some $j$ and the image $\left(r_{j} / r_{i}\right)^{*}$ of $r_{j} / r_{i}$ in $k(w)$ is contained in $R / m$; for if $r_{j} / r_{i}^{*} \in$ $k(w) \backslash k$, then $\left(r_{j} / r_{i}\right)^{*}$ is transcendental over $k$ and hence $\overline{\left(r_{i}, r_{j}\right)} \subseteq J$ by Remark 1.3 above. Therefore, $w\left(r_{i}\right) \geq e$, which is a contradiction to our assumption. We conclude that the image $\left(r_{j} / r_{i}\right)^{*}$ of $r_{j} / r_{i}$ in $k(w)$ is in $R / m$ for all $j$ such that $w\left(r_{j}\right)=w\left(r_{i}\right)$. For all $r_{j}$ such that $w\left(r_{j}\right)>w\left(r_{i}\right), r_{j} / r_{i} \in$ $m(W)$. Hence, $\left(r_{j} / r_{i}\right)^{*} \in k$ for all such $j$. Therefore, $\left(r_{j} / r_{i}\right)^{*} \in k \forall j$. Since $w(f)>w\left(r_{i}\right)$, it follows that $w\left(f / r_{i}\right)>0$. This implies that $f / r_{i} \in m(W)$. Hence,

$$
\left(f / r_{i}\right)^{*}=\sum_{j=0}^{n}\left(r_{j} / r_{i}\right)^{*}(a / b)^{* j} \equiv 0 \quad(\bmod m(W))
$$

in $k(w)$. Since $\left(r_{j} / r_{i}\right)^{*} \in R / m \forall j,(a / b)^{*}$ is algebraic over $R / m$, which contradicts Remark 1.2. Hence, $w(f)=\min \left\{w\left(r_{j}\right)\right\} \in w(R)$. This proves the claim.

Therefore, $w(R)=w(R[a / b])$. Since $J$ is integrally closed, $J$ is also a normal ideal by Zariski's Product Theorem (cf. [ZS, Theorems 2 and 2', Appendix 5]). Hence, $R[J / b]=R[J t][1 /(b t)] \cap K$ is normal, and it is the integral closure of $R[a / b]$. Since $W$ is the Rees valuation ring of $J, W=$ $R[j / b]_{P}$ where $P=\sqrt{J} R[J / b]$ is a height 1 prime. Since $a$ and $b$ are a regular sequence, $R[a / b] \simeq R[X] /(b X-a)$, where $X$ is a variable. Hence, $R[a / b] / m R[a / b] \simeq k[X]$ since $(b X-a) \subseteq m R[X]$. Therefore, $m R[a / b]$ is a height 1 prime in $R[a / b]$. Let $m(W) \cap R[a / b]=Q$. Then $Q \supseteq m R[a / b]$. If 
ht $Q=2$, then

$$
R[a / b] / Q \simeq k[X] / Q^{\prime},
$$

where $Q^{\prime}$ is the image of $Q$ in $k[X]$. Therefore $R[a / b] / Q$ is finite algebraic over $k$ by the Nullstellensatz [AM, Chapter 5]. But $a / b \in R[a / b]$ implies that $(a / b)^{*} \in R[a / b] / Q$, which is a contradiction to Remark 1.2 since $(a / b)^{*}$ is transcendental over $k$. Hence, $m(W) \cap R[a / b]=m R[a / b]$, i.e., $W=\bar{A}$, where $A=R[a / b]_{m R[a / b]}$. On the other hand,

$$
A=R[a / b]_{m R[a / b]} \simeq R(X) /(b X-a),
$$

where $R(X)=R[X]_{m R[X]}, X$ is a variable [N, $\left.\S 6\right]$. Then $R(X)$ is also a 2-dimensional RLR and $(b X-a)$ is a height 1 prime of $R(X)$. Hence, $A$ is a 1-dimensional local complete intersection, i.e., $A$ is a Gorenstein ring. Furthermore, $A / m(A) \simeq k\left(a / b^{*}\right)=k(w)$ and $A$ is analytically irreducible since $\bar{A}=W$ is local [N, $\S 33$, Exercise 1]. By Theorem 1.1 of Kunz, $w(A)$ is symmetric. Therefore $w(R)$ is symmetric.

In [S] Judy Sally defined an ideal $I$ to be a 1-fibered ideal if $I$ has only one Rees valuation. She also asked about a possible generalization of Theorem 1 as follows:

Question 1.6. Let $(R, m, k)$ be a Gorenstein ring with a 1-fibered ideal $I$, and let $v$ be the Rees valuation of $I$. Is $v(R)$ symmetric?

We also have a natural question about generalizing Theorem 1 to higher dimensional RLRs as follows:

Question 1.7. Let $(R, m, k)$ be a 3-dimensional RLR and let $w$ be a prime divisor of $R$ (that is, the valuation ring $W$ dominates $R$ and $\operatorname{trdeg}_{k}(k(w))=$ $2)$. When is $w(R)$ symmetric?

\section{WHEN ARE TWO VALUE SEMIGROUPS EQUAL?}

In this section we compare the value semigroups of two different prime divisors of a 2-dimensional RLR. Before we prove the main theorems we state the definition of the point basis and the base points of an ideal given by Lipman. We refer to [L2] for a more detailed explanation. Even though Lipman has introduced the theory in arbitrary RLRs, we mention it just for 2-dimensional RLRs in this paper. Recall the definition of a first quadratic transform of a 2-dimensional RLR given in the introduction. Let $R \subset S$ be 2-dimensional RLRs with the same quotient field $K$. Then there is always a unique finite sequence of RLRs between $R$ and $S$ as follows [A, Theorem 3]:

$$
R=R_{0} \subset R_{1} \subset R_{2} \subset \cdots \subset R_{n}=S,
$$

where $R_{i}$ is a first quadratic transformation of $R_{i-1}$ for $1 \leq i \leq n$. The above sequence of RLRs is called the quadratic sequence from $R$ to $S$. Let $I$ be a nonzero ideal of $R$. Let $I_{i}$ denote the transform of $I_{i-1}$ in $R_{i}$ for $1 \leq i \leq n$ as defined in the Introduction. Let $I_{S}$ denote the transform of $I$ in $S$ and let $o_{S}$ denote the $m(S)$-adic order valuation. Then the point basis of $I$ is the family of nonnegative integers 


$$
B(I)=\left\{o_{S}\left(I_{S}\right) \mid R \subset S \subset K\right\} .
$$

$S$ is called a base point of $I$ if $o_{S}\left(I_{s}\right) \neq 0$, i.e., $I_{S} \neq S$. Let $P(I)$ denote the set of base points of $I$. If $I$ is an integrally closed $m$-primary ideal, then $P(I)$ is finite [L2, Theorem 3.1].

Our special case is when $v$ and $w$ are associated to two adjacent simple integrally closed ideals. Before we compare their value semigroups, we first compare the point bases of two adjacent simple integrally closed ideals as well as some other properties.

Lemma 2.1. Let $(R, m, k)$ be a 2-dimensional $R L R$. Let $I \supset J$ be adjacent simple integrally closed ideals associated to prime divisors $v$ and $w$ respectively. Then

1. $o(I)=o(J)$;

2. there exists an $a \in J$ which is part of the minimal reductions of $I$ and $J$

3. $B(J)=B(I) \cup\{1\}$ and $P(J)=P(I) \cup\left\{R_{n}\right\}$, where $\left(R_{n}, m_{n}\right)$ is an $R L R$ such that the $m_{n}$-adic order valuation is $w$.

Proof. 1. Since $I \supset J$ are adjacent, $I / J \simeq R / m$, hence $I m \subseteq J$. Therefore, $o(I)+1 \geq o(J) \geq o(I)$, i.e., $o(J)=o(I)$ or $o(J)=o(I)+1$. Suppose $o(J)=o(I)+1$ and $o(I)=r$. Then $\lambda(I: m / I)=r$ and $\lambda(J: m / J)=r+1$ as in the proof of [H, Proposition 2.3]. Since $I \subset J: m \subset I: m$, it follows that $I: m=J: m$. Therefore, $m I \subset m(I: m)=(J: m) \subseteq J$, hence $o(I: m)=r$ and $\mu(I: m)=\lambda(I: m / m(I: m))=r+1$. Hence, $J=m(I: m)=m(J: m)$, i.e., $m \mid J$, which is a contradiction since $J$ is simple (cf. [ZS, Proposition 3, Appendix 5]).

2. We can choose $a \in J$ such that $v(a)=v(J)$ and $w(a)=w(J)$. Since $\lambda(I / J)=1$, for a minimal reduction $(c, d)$ of $I$, we may assume that either $c$ or $d$ is contained in $J$. For if we suppose $d \notin J$, then $I=(J, d)$. Hence, $c$ can be written as $j+r d$ for some $j \in J$ and $r \in R$. Replace $c$ by $c-r d$. Suppose $c \in J$. Then $v(c) \geq v(a)=v(J) \geq v(I)=v(c)$, hence $v(a)=v(I)$. Hence $a$ is part of the minimal reductions for $I$ and $J$ (cf. [No, Lemma 1.9]).

3. Let $o(I)=o(J)=r$. Let $a \in J$ be an element of order $r$ which is part of the minimal reductions for $I$ and $J$, and let $(a, b)$ be a minimal reduction of $I$. Then $b \notin J$ since $I$ is not integral over $J$. Since $I$ and $J$ are contracted ideals, $\mu(I)=\mu(J)=r+1[\mathrm{H}$, Proposition 2.3; HS, Theorem 2.1]. Now we choose a basis $\left(a_{1}, \ldots, a_{r}=a, a_{r+1}=b\right)$ for $I$ so that $a_{1}, \ldots, a_{r} \subseteq J$. Then $J / I m=\left(a_{1}^{\prime}, \ldots, a_{r}^{\prime}\right)$, where $a_{1}^{\prime}, \ldots, a_{r}^{\prime}$ are the images of $a_{1}, \ldots, a_{r}$ in $R / I m$ since $\lambda(J / m I)=\mu(I)-\lambda(I / J)=r$. We also choose a basis $(x, y)$ for $m$ so that $(a, x)$ and $(a, y)$ are $m$-primary. We may also assume $x^{*} \nmid a^{*}$, where $x^{*}, y^{*}$, and $a^{*}$ are the leading forms of $x, y$, and $a$ in $\operatorname{gr}_{m}(R)$; for if $x^{*} \mid a^{*}$, then since $k$ is infinite, by replacing $x$ by $x-\alpha y$ for some $\alpha \in k$, we may assume that $x^{*} \nmid a^{*}$ (and we still have that $(a, x)$ is $m$-primary).

With such an $x$ we show that $b x \notin m J$. Since $J$ is a $w$-ideal and $b \notin J$, it follows that $w(b)<w(J)$. Since $J$ is simple, $m \nmid J$; and since $a \in J$ and $o(a)=r$, we have $c(J)=a^{*}$. Thus, $J$ is contracted from $R[m / x]$ by the proof of Proposition 2.3 in [H]. Therefore, $w(y) \geq w(x)$, i.e., $w(m)=w(x)$. Hence, $w(b x)=w(b)+w(x)=w(b)+w(m)<w(J)+w(m)=w(J m)$ implies 
that $b x \notin m J$. We show that we may also assume that $b y \in J m$. Since

$$
\begin{aligned}
\lambda(m I / m J) & =\lambda(I / m J)-\lambda(I / m I)=\lambda(I / J)+\lambda(J / m J)-\lambda(I / m I) \\
& =1+(r+1)-(r+1)=1,
\end{aligned}
$$

and since $b x \notin m J$ it follows that

$$
b y^{\prime}=f^{\prime}\left(b x^{\prime}\right) \text { for some } f^{\prime} \in R / m\left(a_{1}, \ldots, a_{r}\right) \text {. }
$$

Let $\bar{y}=y-f x$, where $f$ is a preimage of $f^{\prime}$. Then $b \bar{y}=b y-b f x \in$ $m\left(a_{1}, \ldots, a_{r}\right) \subseteq m J$. Replace $y$ by $\bar{y}$ so that $b y \in m J$.

Since $a \in I$ and $I$ is simple, we also have $c(I)=a^{*}$, i.e., $x^{*} \nmid c(I)$. Therefore, $I$ is also contracted from $R[m / x]$. Since $w(b)<w(J)$ and $b y \in$ $J m$, we have

$$
w(b)+w(y) \geq w(J)+w(m)>w(b)+w(m) .
$$

Hence, $w(y)>w(m)$, and so $w(y)>w(x)$. Since $J$ is simple, the transform $J^{\prime}$ of $J$ in $R[\mathrm{~m} / x]$ is also simple and contained in a unique maximal ideal $N=(m, y / x)$ in $R[m / x]$ [ZS, p. 386, Lemma 6; H, Remark 3.8]. Since $o(I)=$ $r$, the transform $I^{\prime}$ of $I$ in $R[m / x]$ is $I / x^{r}=\left(a_{1}, \ldots, a_{r}, b\right) / x^{r}$. Hence, $J^{\prime} \subset I^{\prime} \subseteq N=(x, y / x)$ in $R[m / x]$. Let $R_{1}=R[m / x]_{N}, m_{1}=\max \left(R_{1}\right)$, $I_{1}=$ the transform of $I$ in $R_{1}$, and $J_{1}=$ the transform of $J$ in $R_{1}$. Then $I_{1}$ and $J_{1}$ are simple integrally closed ideals of $R_{1}$. Since $b y \in m J$, we have

$$
\left(b / x^{r}\right)(y / x)=b y / x^{r+1} \in J_{1} \text {. }
$$

Hence, $m_{1} I_{1} \subseteq J_{1}$. Therefore $\lambda\left(I_{1} / J_{1}\right)=1$, i.e., $I_{1}$ and $J_{1}$ are adjacent in $R_{1}$. Furthermore, $o_{1}\left(I_{1}\right)=o_{1}\left(J_{1}\right)$ by Lemma 2.1.1, where $o_{1}$ is the $m_{1}$-adic order valuation. By the same argument we can see that $I$ and $J$ go along the same quadratic sequence

$$
\left(R_{0}, m_{0}\right) \subset\left(R_{1}, m_{1}\right) \subset \cdots \subset\left(R_{n}, m_{n}\right),
$$

where $w$ is the $m_{n}$-adic order valuation. Let $I_{i}=$ the ideal transform of $I$ in $R_{i}, J_{i}=$ the ideal transform of $J$ in $R_{i}, m_{i}=\max \left(R_{i}\right)$, and $o_{i}=$ the $m_{i}$-adic order valuation for $i=0,1, \ldots, n$. Then $\lambda\left(I_{i} / J_{i}\right)=1$ and $o_{i}\left(I_{i}\right)=o_{i}\left(J_{i}\right)$ for $i=0,1, \ldots, n-1$. Since $J_{n}=m_{n}, J_{n-1}$ must be an ideal right below $m_{n-1}$, i.e., $I_{n-1}=m_{n-1}$. [Proof: If $R_{1}=R[y / x]_{m R[y / x]}$ is a first quadratic transformation of $R$ with $m=(x, y)$ and the transform $I^{\prime}$ of a $v$-ideal $I$ (for some prime divisor $V$ such that $R \subset R_{1} \subset V$ ) is the maximal ideal of $R_{1}$, then the inverse transform $I^{\prime \prime}$ of $I^{\prime}$ is $x(x, y / x) R_{1} \cap R=\left(x^{2}, y\right)$. Since $I$ is a $v$-ideal, by [ZS, (D.3), p. 390], $I$ is the inverse transform of a $v$-ideal $K$ in $R_{1}$. Since the transform of the inverse transform of $K$ is $K$ itself, we have $I^{\prime}=K=m_{1}$. Since $I$ and $I^{\prime \prime}$ are simple $v$-ideals with the same transforms in $R_{1}, I=I^{\prime \prime}$ by [ZS, (D.4), p. 390], i.e., $I$ is the $v$ ideal right below the maximal ideal $m$.] Hence, $I_{n}=R_{n}$. Therefore $P(I)=$ $\left\{R_{0}, R_{1}, \ldots, R_{n-1}\right\}$ and $P(J)=\left\{R_{0}, R_{1}, \ldots, R_{n}\right\}$, i.e., $P(J)=P(I) \cup\left\{R_{n}\right\}$. Also $B(I)=\left\{o_{i}\left(I_{i}\right)\right\}_{i=0}^{n-1}$ and $B(J)=\left\{o_{i}\left(J_{i}\right)\right\}_{i=0}^{n}$, i.e., $B(J)=B(I) \cup\{1\}$.

We have only proved Lemma 2.1 for simple adjacent integrally closed ideals $I$ and $J$. All three statements in Lemma 2.1 can be proved more generally .

Now let us discuss the value semigroups of 1-dimensional analytically irreducible Gorenstein rings. Let $(A, m)$ be a 1-dimensional analytically irreducible Gorenstein domain. Then $\bar{A}$ is local, i.e., a DVR, and $\lambda(\bar{A} / A)<\infty$. 
Let $v$ be the corresponding valuation of $\bar{A}$. Let $m=\left(a_{1}, \ldots, a_{n}\right)$ and $v(m)=v\left(a_{1}\right)$. Set $b_{i}=a_{i} / a_{1}, B=A\left[b_{1}, \ldots, b_{n}\right]$, and $P=m(\bar{A}) \cap B$. Then $B_{P}$ dominates $A$ and $B_{P} \subseteq \bar{A} ; B_{P}$ is called a first quadratic transformation of $A$ with respect to $\bar{A}$. Since $\lambda(\bar{A} / A)<\infty$, there is a unique finite sequence of quadratic transformations from $A$ along $\bar{A}$ as follows [N, §38]:

$$
A=A_{0} \subset A_{1} \subset \cdots \subset A_{s}=\bar{A} .
$$

Let $e_{i}=e\left(A_{i}\right)$. Then $\left\{e_{0}, e_{1}, \ldots, e_{s}\right\}$ is called the multiplicity sequence of $A$. Note that $e\left(A_{i}\right)=v\left(m\left(A_{i}\right)\right)$ for each $i$.

A plane algebroid curve over an algebraically closed field $k$ of characteristic 0 is a ring of the form $A=k[[x(t), y(t)]]$, i.e., a 1-dimensional complete local domain such that $m(A)=(x(t), y(t))$ and $A=k+m(A)$. In [Az] the multiplicity sequence of such a curve $A$ is discussed and used to study the value semigroup of $A$. Azevedo has many other descriptions of the value semigroups of plane algebroid curves in [Az]; we introduce one that compares the value semigroups of two plane algebroid curves as follows:

Theorem 2.2 (Azevedo [Az]). Let $A$ and $B$ be plane algebroid curves. Then $A$ and $B$ have the same value semigroups if and only if $A$ and $B$ have the same multiplicity sequences.

Below we connect the point basis theory of Lipman to the theory of plane algebroid curves of Azevedo, so that we obtain a nice comparison of two prime divisors of a 2-dimensional RLR. In the proof of Theorem 2, we first show that the point basis of a simple integrally closed ideal gives the multiplicity sequence of a 1-dimensional analytically irreducible local domain which is a Gorenstein ring.

Proof of Theorem 2. By Lemma 2.1, the quadratic sequence along the valuation ring $V$ is part of the quadratic sequence along the valuation ring $W$. Let

$$
\left(R=R_{0}, m=m_{0}\right) \subset\left(R_{1}, m_{1}\right) \subset \cdots \subset\left(R_{n+1}, m_{n+1}\right)
$$

be the quadratic sequence along $W$, where

$$
\begin{aligned}
v & =m_{n} \text {-adic order valuation, } \\
w & =m_{n+1} \text {-adic order valuation, } \\
o_{i} & =m_{i} \text {-adic order valuation, } \\
I_{i} & =\text { ideal transform of } I \text { in } R_{i}, \\
J_{i} & =\text { ideal transform of } J \text { in } R_{i} .
\end{aligned}
$$

Since $I$ and $J$ are simple integrally closed ideals, $I_{i}$ and $J_{i}$ are also simple integrally closed ideals for each $i$. Choose $a$ in $J$ so that $a$ is part of a minimal reduction of $I$ and $J$ as in the proof of Lemma 2.1. Let $(a, b)$ and $(a, c)$ be minimal reductions of $I$ and $J$ respectively. Then $o_{i}\left(I_{i}\right)=o_{i}\left(J_{i}\right)$ for $0 \leq i \leq n$ by Lemma 2.1; let $o_{i}\left(I_{i}\right)=r_{i}$. By Theorem 1.4, $o_{i}\left(I_{i}\right)=$ $v\left(m_{i}\right)$ for each $i$. Therefore $r_{0} \geq r_{1} \geq \cdots \geq r_{n}$ since $m_{i} \cap R_{i-1}=m_{i-1}$ for $1 \leq i \leq n$. Let $N$ be the maximal ideal in $R[m / x]$ for which $R_{1}=$ $R[m / x]_{N}$. Choose generators $x, y$ of $m$ so that $x^{*} \nmid a^{*}$. Let $a_{1}=a / x^{r}$, $b_{1}=b / x^{r}$, and $c_{1}=c / x^{r}$. Then $\left(a_{1}, b_{1}\right)$ is a minimal reduction of $I_{1}$ and $\left(a_{1}, c_{1}\right)$ is a minimal reduction of $J_{1}$. In the same way we obtain $a_{i}, b_{i}, c_{i}$ 
for $i=0, \ldots, n$. Then $I_{n}=\left(a_{n}, b_{n}\right)=m_{n}$ and $J_{n+1}=\left(a_{n+1}, c_{n+1}\right)=m_{n+1}$. [Proof: Since $m_{n}=\overline{\left(a_{n}, b_{n}\right)}$, it follows that $e\left(m_{n}\right)=e\left(\left(a_{n}, b_{n}\right)\right)$. Since $R_{n}$ is regular, it is Cohen-Macaulay. Hence, $e\left(m_{n}\right)=e\left(\left(a_{n}, b_{n}\right)\right)=\lambda\left(R_{n} /\left(a_{n}, b_{n}\right)\right)$ by Theorem 17.11 in [Ma]. Since $R_{n}$ is regular, $e\left(m_{n}\right)=1$ [N, (40.6)]. Therefore, $\lambda\left(R_{n} /\left(a_{n}, b_{n}\right)\right)=1$, i.e., $\left(a_{n}, b_{n}\right)=m_{n}$. By the same token, we have that $\left.m_{n+1}=\left(a_{n+1}, c_{n+1}\right).\right]$

Let

$$
A_{i}=R_{i}\left[b_{i} / a_{i}\right]_{m_{i} R_{i}\left[b_{i} / a_{i}\right]} \simeq R_{i}(X) /\left(a_{i} X-b_{i}\right)
$$

for $i=0, \ldots, n$. Then, since the order of the element $a_{i} X-b_{i}$ in the 2dimensional RLR $R_{i}(X)$ is $o_{i}\left(a_{i}, b_{i}\right)$, we get $e\left(A_{i}\right)=e\left(m\left(A_{i}\right)\right)=o_{i}\left(a_{i}, b_{i}\right)$ by (40.2) in [N]. Since $\left(a_{i}, b_{i}\right)$ is a minimal reduction of $I_{i}, o_{i}\left(a_{i}, b_{i}\right)=o_{i}\left(I_{i}\right)=r_{i}$ for $0 \leq i \leq n$. Suppose $t$ is the largest number such that $r_{t-1}>1$ and $r_{i}=1$ for $t \leq i \leq n$. Then $o_{t}\left(I_{t}\right)=o_{t}\left(a_{t}, b_{t}\right)=1$, and therefore

$$
A_{t}=R_{t}\left[b_{t} / a_{t}\right]_{m_{t} R_{t}\left[b_{t} / a_{t}\right]} \simeq R_{t}(X) /\left(a_{t} X-b_{t}\right)
$$

is a DVR since $R_{t}(X)$ is an RLR and $a_{t} X-b_{t}$ is part of a regular system of parameters of $R_{t}(X)$ (cf. [N, (25.18)]). Therefore, $A_{t}=\bar{A}=V$ and

$$
A=A_{0} \subset A_{1} \subset \cdots \subset A_{t}=\bar{A}=V
$$

is the quadratic sequence of $A$ along $\bar{A}$ with $e\left(A_{i}\right)=v\left(m\left(A_{i}\right)\right)=r_{i}$ for $0 \leq i \leq t$. Therefore, $\left\{r_{0}, r_{1}, \ldots, r_{t}\right\}$ is the multiplicity sequence of a 1dimensional Gorenstein domain $A=R[b / a]_{m R[b / a]}$.

Similarly, let

$$
B_{i}=R_{i}\left[c_{i} / a_{i}\right]_{m_{i} R_{i}\left[c_{i} / a_{i}\right]} \simeq R_{i}(X) /\left(a_{i} X-c_{i}\right)
$$

for $i=0, \ldots, n+1$. Let $t$ be the number defined as above. Then

$$
B_{t}=R_{t}\left[c_{t} / a_{t}\right]_{m_{t} R_{t}\left[c_{t} / a_{t}\right]} \simeq R_{t}(X) /\left(a_{t} X-c_{t}\right)=W
$$

by the same argument as above. Therefore,

$$
B=B_{0} \subset B_{1} \subset \cdots \subset B_{t}=\bar{B}=W
$$

is the quadratic sequence of $B$ along $\bar{B}$ and $e\left(B_{i}\right)=o_{i}\left(a_{i}, c_{i}\right)=o_{i}\left(J_{i}\right)$ for $0 \leq i \leq t$. Since $o_{i}\left(I_{i}\right)=o_{i}\left(J_{i}\right)=r_{i}$, it follows that $e\left(A_{i}\right)=e\left(B_{i}\right)$ for $0 \leq i \leq t$. Therefore $\left\{r_{0}, r_{1}, \ldots, r_{t}\right\}$ is also the multiplicity sequence of a 1-dimensional Gorenstein domain $B=R[c / a]_{m R[c / a]}$.

In the proof of Theorem 1, we showed that $v(R)=v(A)$ and $w(R)=w(B)$. Hence, it is enough to see that $v(A)=w(B)$. Let $\hat{A}_{i}$ be the $m\left(A_{i}\right)$-adic completion of $A_{i}$ for $i=0,1, \ldots, t$. Since $m_{i} R_{i}\left[b_{i} / a_{i}\right]$ is a prime ideal of $R_{i}\left[b_{i} / a_{i}\right], \widehat{A_{i}}=\widehat{R}_{i}\left[b_{i} / a_{i}\right]_{\widehat{m}_{i} \widehat{R}_{i}\left[b_{i} / a_{i}\right]}$. Since $R$ is an RLR, $\widehat{R}$ is a domain, hence so is $\widehat{A}$. Since $\widehat{R}_{i} \subset$ q.f. $(\widehat{R})$ and $b_{i} / a_{i}=b / a \in$ q.f. $(\widehat{A})$, we have the containment

$$
\hat{A} \subset \widehat{A_{1}} \subset \widehat{A_{2}} \subset \cdots \subset \widehat{A_{t}}=\widehat{V}
$$

in q.f. $(\widehat{A})$. Therefore $\overline{\hat{A}}$ is a DVR [N, §33]. However $\widehat{V}$ is also a DVR since it is a 1-dimensional Noetherian domain with the principal maximal ideal $m(V) \widehat{V}$. Since $\widehat{A} \subset \widehat{V}$, it follows that $\bar{A} \subseteq \widehat{V} \subset$ q.f. $(\widehat{A})$, which implies $\overline{\widehat{A}}=\widehat{V}$. Furthermore,

$$
\widehat{A} \subset \widehat{A_{1}} \subset \cdots \subset \widehat{A_{t}}=\widehat{V}=\overline{\widehat{A}}
$$


is the sequence of the quadratic transformations of $\widehat{A}$ along its integral closure with the multiplicity sequence $\left\{r_{0}, r_{1}, \ldots, r_{t}\right\}$. Similarly,

$$
\widehat{B} \subset \widehat{B}_{1} \subset \cdots \subset \widehat{B}_{t}=\widehat{W}=\overline{\widehat{B}}
$$

is the sequence of quadratic transformations of $\widehat{B}$ along its integral closure with the multiplicity sequence $\left\{r_{0}, r_{1}, \ldots, r_{t}\right\}$. Let $\hat{v}$ and $\widehat{w}$ be the valuations of $\widehat{V}$ and $\widehat{W}$ respectively. Then $\hat{v}(\widehat{A})=\widehat{w}(\widehat{B})$ by Theorem 2.2 since they have the same multiplicity sequences. But $\hat{v}(\widehat{A})=v(A)$ and $\widehat{w}(\widehat{B})=w(B)$; therefore $v(R)=v(A)=w(B)=w(R)$, i.e., $v$ and $w$ have the same value semigroups on $R$.

We also can ask about the converse of Theorem 2, i.e., if $w$ and $v$ have the same value groups on $R$, then are they associated to adjacent simple integrally closed ideals? The answer is no, and $J$ does not even have to be contained in $I$, as Theorem 3 shows.

Proof of Theorem 3. Suppose that $v(R)=w(R)$. Let $(a, b)$ be a minimal reduction of $I$ and $(c, d)$ be a minimal reduction of $J$. Let $A=R[a / b]_{m R[a / b]}$ and $B=R[c / d]_{m R[c / d]}$, and let $\widehat{A}$ and $\widehat{B}$ be the completions of $A$ and $B$ respectively. In the proof of Theorem 2 we showed that $\bar{A}=\widehat{V}$ and $\overline{\widehat{B}}=\widehat{W}$. Let $\hat{v}$ and $\widehat{w}$ be the corresponding discrete valuations of $\widehat{V}$ and $\widehat{W}$. Both $\widehat{w}$ and $\hat{v}$ are valuations of the same field, which is the quotient field of both $\widehat{A}$ and $\widehat{B}$. In Theorem 2 we saw that $\hat{v}(\widehat{A})=\widehat{w}(\widehat{B})$. Therefore, the multiplicity sequences of $\widehat{A}$ and $\widehat{B}$ are equal by Theorem 2.2. Therefore, $n=h$ and $s_{i}=t_{i} \quad \forall i=0,1, \ldots, n$. Now let us prove the converse. We showed that $\left\{s_{0}, s_{1}, \ldots, s_{n}\right\}$ and $\left\{t_{0}, t_{1}, \ldots, t_{h}\right\}$ are the multiplicity sequences of $\widehat{A}$ and $\widehat{B}$ in Theorem 2. Since $n=h$ and $s_{i}=t_{i}$ for all $i=0,1, \ldots, n$, it follows that $\hat{v}(\widehat{A})=\widehat{w}(\widehat{B})$ by Theorem 2.2. Therefore, $v(A)=w(B)$, and furthermore $v(R)=w(R)$ by the proof of Theorem 1 .

Remark 2.3. In [L3], Lipman gives a formula for the conductor element of $w$ associated to a simple integrally closed ideal $J$ as follows:

$$
c(w)=\sum r_{i}\left(r_{i}-1\right),
$$

where $r_{i} \in B(J)$. Therefore for two prime divisors $v$ and $w$ which are associated to adjacent simple integrally closed ideals $I$ and $J, c(v)=c(w)$ since $B(J)=B(I) \cup\{1\}$.

Example 2.4 [Hos, p. 70]. Let $m=(x, y), I=\left(x^{3}, x^{2} y, y^{2}\right)$, and $J=$ $\left(y^{2}-x^{3}, x^{2} y, x^{4}\right)$. Then $I$ and $J$ are adjacent simple integrally closed ideals. Let $v$ and $w$ be the prime divisors associated to $I$ and $J$ respectively. Then the quadratic sequence of $R$ along $w$ is as follows:

$$
\begin{array}{cc}
R_{1}=R[m / x]_{(x, y / x)}, & m_{1}=\left(x_{1}=x, y_{1}=y / x\right), \\
R_{2}=R_{1}\left[m_{1} / y_{1}\right]_{\left(y_{1}, x_{1} / y_{1}\right)}, & m_{2}=\left(x_{2}=x^{2} / y, y_{2}=y / x\right), \\
R_{3}=R_{2}\left[m_{2} / x_{2}\right]_{\left(x_{2}, y_{2} / x_{2}\right)}, & m_{3}=\left(x_{3}=x^{2} / y, y_{3}=y^{2}-x^{3} / x^{3}\right) .
\end{array}
$$


The ideal transforms of $I$ and $J$ are as follows:

$$
\begin{aligned}
& I_{1}=\left(x_{1}, y_{1}^{2}\right)=\left(x, y^{2} / x^{2}\right), \\
& I_{2}=m_{2}=\left(x^{2} / y, y / x\right), \\
& J_{1}=\left(y_{1}^{2}-x_{1}=y^{2}-x^{3} / x^{2}, y_{1} x_{1}=y\right), \\
& J_{2}=\left(y_{2}-x_{2}, x_{2}^{2}\right)=\left(y^{2}-x^{3} / x y, x^{4} / y^{2}\right), \\
& J_{3}=m_{3}=\left(x^{2} / y, y^{2}-x^{3} / x^{3}\right) .
\end{aligned}
$$

Therefore, $v(x)=2, v(y)=3$ and $w(x)=2, w(y)=3, w\left(y^{2}-x^{3}\right)=7$. Since $v(I)=v(J)$, we have $v\left(y^{2}-x^{3}\right)=6$. Furthermore, $B(I)=\{2,1,1\}$, $B(J)=\{2,1,1,1\}$, and $v(R)=w(R)=\{0,2,3,4, \ldots\}$, a symmetric value semigroup with the conductor element 2 .

Example 2.5 [Hos, p. 70]. We just proved that two prime divisors $v$ and $w$ associated to adjacent simple integrally closed ideals $I$ and $J$ have the same value semigroups on $R$. However, if $I$ is not simple, it may be that neither of the valuations associated to the factors of $I$ have the same value semigroup as $w$; and the product of the semigroups for these valuations need not be the value semigroup of $w$. For example, if $I=\left(x^{3}, x^{2} y, y^{2}\right)\left(y^{2}-x^{3}, x^{2}, x^{4}\right)$ and $J=\left(\left(y^{2}-x^{3}\right)^{2}, x y^{4}, x^{2} y^{3}, x^{5} y, x^{7}\right)$, then $I$ and $J$ are adjacent integrally closed ideals. Let $v_{1}$ and $v_{2}$ be the prime divisors corresponding to $\left(x^{3}, x^{2} y, y^{2}\right)$ and $\left(y^{2}-x^{3}, x^{2}, x^{4}\right)$ respectively. In Example 2.4 we saw that $c\left(v_{1}\right)=c\left(v_{2}\right)=2$ and $v_{1}(R)=v_{2}(R)=\{0,2,3, \ldots\}$. Let $w$ be the prime divisor associated to $J$. Then $B(J)=\{4,2,2,1,1\}, c(w)=16$, and $w(R)=\{0,4,6,8,10,12,13,14,16,17,18, \ldots\}$. That is, $v_{i}(R) \neq w(R)$ for $i=1,2$ in this case.

\section{ACKNOWLEDGMENTS}

My sincere thanks are due to Professors W. Heinzer and C. Huneke for introducing and teaching me this subject with warm encouragement. Special thanks are also due to Professors J. Ratliff, D. Rush, and K. Shah for their helpful comments. I am also grateful to the referee for valuable comments.

\section{REFERENCES}

[A] S. S. Abhyankar, On the valuations centered in a local domain, Amer. J. Math. 78 (1956), 321-348.

[AM] M. F. Atiyah and I. G. MacDonald, Introduction to commutative algebra, Addison-Wesley, 1969.

[Az] A. Azevedo, Lecture on plane algebroid curve at Purdue University, 1980

[Hos] M. A. Hoskin, Zero-dimensional valuation ideals associated with plane curves branches, Proc. London Math. Soc. (3) 6 (1956), 70-99.

[H] C. Huneke, Complete ideals in two-dimensional regular local rings, Microprogram in Commutative Algebra, MSRI, Springer-Verlag, 1987.

[HS] C. Huneke and J. D. Sally, Birational extensions in dimension two and integrally closed ideals, J. Algebra 115 (1988), 481-500.

[K1] E. Kunz, The value-semigroup of a one dimensional Gorenstein ring, Proc. Amer. Math. Soc. 25 (1970), 748-751.

[K2] _ Introduction to commutative algebra and algebraic geometry, Birkhäuser, 1985. 
[L1] J. Lipman, Rational singularities, with applications to algebraic surfaces and unique factorization, Inst. Hautes Études Sci. Publ. Math. 36 (1969), 195-279.

[L2] _ On complete ideals in regular local rings, Algebraic Geometry and Commutative Algebra in Honor of Masayoshi Nagata, Vol. I, Academic Press, 1988, pp. 203-231.

[L3] Handwritten notes, 1966.

[M] S. McAdam, Asymptotic prime divisors, Lecture Notes in Math., vol. 1023, Springer-Verlag, 1983.

[Ma] H. Matsumura, Commutative ring theory, Cambridge Univ. Press, 1986.

[N] M. Nagata, Local rings, Interscience, New York, 1962.

[No] S. Noh, Sequence of valuation ideals of prime divisors of the second kind in 2-dimensional regular local rings, J. Algebra (to appear).

[R] D. Rees, Lectures on the asymptotic theory of ideals, London Math. Soc. Lecture Note Series, vol. 113, Cambridge Univ. Press, 1988.

[S] J. Sally, One-fibered ideals, Microprogram in Commutative Algebra, MSRI, Springer-Verlag, 1987.

[Z] O. Zariski, Polynomial ideals defined by infinitely near base points, Amer. J. Math. 60 91938), 151-204.

[ZS] O. Zariski and P. Samuel, Commutative algebra, Vol. 2, Van Nostrand Princeton, N.J., 1960.

Department of Mathematics, University of California, Riverside, California 92521 08903

Current address: Department of Mathematics, Rutgers University, New Brunswick, New Jersey

E-mail address: noh@math.rutgers.edu 